\title{
Platelet Engraftment
}

National Cancer Institute

\section{Source}

National Cancer Institute. Platelet Engraftment. NCI Thesaurus. Code C63323.

A platelet count of 20,000 to 50,000 (platelets per microliter of blood) is a sign of platelet eng raftment. For patients who receive marrow or peripheral blood cells, platelet engraftment often happens at the same time or a little bit after neutrophil engraftment. For patients who receive cord blood, it may be 8 weeks or longer after transplant before platelet engraftment occurs. 